ARTICLE

Received 2 Nov 2015 | Accepted 9 Nov 2016 | Published 20 Dec 2016

DOI: $10.1038 /$ ncomms13884

OPEN

\title{
MicroRNA-182 targets SMAD7 to potentiate TGF $\beta$-induced epithelial-mesenchymal transition and metastasis of cancer cells
}

Jingyi Yu ${ }^{1, \star}$, Rong Lei ${ }^{1, \star}$, Xueqian Zhuang ${ }^{1}$, Xiaoxun $\mathrm{Li}^{1}$, Gang Li ${ }^{1}$, Sima Lev ${ }^{2}$, Miguel F. Segura ${ }^{3, \dagger}$, Xue Zhang ${ }^{1} \&$ Guohong $\mathrm{Hu}^{1}$

The transforming growth factor $\beta$ (TGF $\beta$ ) pathway plays critical roles during cancer cell epithelial-mesenchymal transition (EMT) and metastasis. SMAD7 is both a transcriptional target and a negative regulator of TGF $\beta$ signalling, thus mediating a negative feedback loop that may potentially restrain TGF $\beta$ responses of cancer cells. Here, however, we show that TGF $\beta$ treatment induces SMAD7 transcription but not its protein level in a panel of cancer cells. Mechanistic studies reveal that TGF $\beta$ activates the expression of microRNA-182 (miR-182), which suppresses SMAD7 protein. miR-182 silencing leads to SMAD7 upregulation on TGF $\beta$ treatment and prevents TGF $\beta$-induced EMT and invasion of cancer cells. Overexpression of miR-182 promotes breast tumour invasion and TGF $\beta$-induced osteoclastogenesis for bone metastasis. Furthermore, miR-182 expression inversely correlates with SMAD7 protein in human tumour samples. Therefore, our data reveal the miR-182mediated disruption of TGF $\beta$ self-restraint and provide a mechanism to explain the unleashed TGF $\beta$ responses in metastatic cancer cells.

\footnotetext{
${ }^{1}$ The Key Laboratory of Stem Cell Biology, Institute of Health Sciences, Shanghai Institutes for Biological Sciences, Chinese Academy of Sciences \& Shanghai Jiao Tong University School of Medicine, University of Chinese Academy of Sciences, Shanghai 200031, China. ${ }^{2}$ Molecular Cell Biology Department, Weizmann Institute of Science, Rehovot 76100, Israel. ${ }^{3}$ Department of Pathology, New York University School of Medicine, New York, New York 10016, USA. ${ }^{\star}$ These authors contributed equally to this work. † Present address: Laboratory of Translational Research in Adolescent and Childhood Cancer, Vall d'Hebron Research Institute (VHIR)-UAB, Barcelona 119129-08035, Spain. Correspondence and requests for materials should be addressed to G.H.

(email: ghhu@sibs.ac.cn).
} 
T he TGF $\beta$ pathway is a key player in embryonic development and cellular homoeostasis in species ranging from flies to mammals. The signalling cascade initiates when the ligands bind to TGF $\beta$ type II receptors, which recruit and phosphorylate type I receptors. The type I receptors in turn phosphorylate the receptor-regulated SMADs (R-SMADs), SMAD2 and SMAD3, that form complexes with the common SMAD (Co-SMAD) protein SMAD4 and shuttle into the nucleus. $\mathrm{R}-\mathrm{SMAD} / \mathrm{co}-\mathrm{SMAD}$ complexes accumulate in the nucleus and bind to target genes for transcriptional regulation ${ }^{1,2}$. The malfunction of TGF $\beta$ signalling can result in many pathological changes, among which epithelial-mesenchymal transition (EMT) is a well-studied process that endows cancer cells with increased aggressiveness. EMT refers to the reprogramming of epithelial cells to a mesenchymal-like phenotype, which occurs in many developmental processes such as gastrulation, neurulation and heart morphogenesis ${ }^{3,4}$. The process is driven by a set of transcriptional factors, including the zinc finger factors Snail, Slug, ZEB1/2 and FOXC2, and the basic helix-loop-helix factors TWIST and E47. These factors coordinate in an elaborate manner to suppress the expression of the epithelial marker E-cadherin (CDH1) and induce the expression of mesenchymal markers such as N-cadherin (CDH2), Vimentin and Fibronectin. The TGF $\beta$ pathway regulates, acting alone or in cooperation with other signalling pathways, these transcription factors, which confers TGF $\beta$ a potent inducer of EMT (refs 5,6). As a physiological phenomenon hijacked by cancer, EMT enhances cancer cell stemness, motility and invasiveness ${ }^{7,8}$. In addition to EMT, TGF $\beta$ signalling plays roles in other metastasis-related processes, including microenvironment remodelling of target organs for cancer cell metastatic outgrowth. In particular, TGF $\beta$ is critical for cancer cell adaptation and colonization of bone. After arriving at bone, cancer cell responds to TGF $\beta$ stimulation and promotes osteoclast maturation via secretory factors such as PTHLH. In turn mature osteoclasts cause bone digestion, leading to the release of various growth factors including TGF $\beta$ embedded in bone matrix and thus further stimulation of cancer cells, constituting so called osteolytic 'vicious cycle ${ }^{99,10}$. Thus TGF $\beta$ responsiveness is a prerequisite of cancer cells for initiating osteolytic metastasis ${ }^{9,11,12}$.

Since TGF $\beta$ signalling has vital roles in multiple biological processes, the pathway components, including the ligands, the receptors and the SMAD proteins, are tightly regulated by various mechanisms. One such mechanism is mediated by the inhibitory SMAD (I-SMAD) protein SMAD7. SMAD7 can compete with R-SMADs for binding to the type I receptor and prevents their phosphorylation ${ }^{1,13}$. It can also recruit SMURF to TGF $\beta$ receptors for polyubiquitination and degradative endocytosis ${ }^{14}$. In addition, SMAD7 disrupts the formation of TGF $\beta$-induced SMAD-DNA complex by binding to the SMAD-binding elements (SBE) via its MH2 domain ${ }^{15}$. As SMAD7 transcription is rapidly induced by TGF $\beta$ (refs 13,16), it represents a negative feedback mechanism for precise control of cellular responses to TGF $\beta$, which is critical during physiological regulation. However, it is unclear whether or how this feedback loop can be disrupted under pathological conditions, especially in cancer cells.

MicroRNAs (miRNAs) are small non-coding RNAs of 19-24 nucleotides in length and exert their regulatory functions by mRNA degradation or translational inhibition. Accumulating evidence demonstrates that miRNAs play critical roles in TGF $\beta$ signalling regulation, EMT and cancer metastasis ${ }^{17,18}$. Among them, miR-182 has been recently found to promote cancer cell metastasis and also mediate the crosstalk between TGF $\beta$ and NFKB pathways ${ }^{19}$. In this study, we report a new role of miR-182 to potentiate TGF $\beta$ signalling. miR-182 is induced by TGF $\beta$ and targets SMAD7 for translational inhibition. The expression of
miR-182 antagonizes the response of SMAD7 to TGF $\beta$ and promotes cancer cell EMT, invasion, as well as distant metastasis. Therefore, our study reveals a novel mechanism of cancer cells to disengage the negative feedback chain of TGF $\beta$ during metastasis.

\section{Results}

TGF $\beta$ activates SMAD7 transcription but not translation. TGF $\beta$ stimulation usually elicits sustained morphological and behavioural changes of cancer cells, despite the SMAD7-mediated negative feedback of the pathway. To investigate whether the feedback loop actually takes effect in cancer cells, we treated the breast cancer cell line MCF10AT with the TGF $\beta 1$ cytokine in a time course of 6-72 $\mathrm{h}$, and then examined the SMAD7 expression. As expected, the SMAD7 mRNA was rapidly induced by TGF $\beta$ and sustained at high levels during the whole time course. However, when the SMAD7 protein was analysed, it was scantily increased at first but then gradually dropped after $6 \mathrm{~h}$, a pattern in stark contrast to the mRNA changes (Fig. 1a). This phenomenon was also observed in an additional panel of breast and lung cancer cell lines, including SCP28 (ref. 20), MCF10AT, 4T1, EpRas and A549. After TGF $\beta$ treatment for $72 \mathrm{~h}$, SMAD7 mRNA was significantly induced, but the protein was kept unchanged or even slightly down-regulated (Fig. 1b). Meanwhile, TGF $\beta$ treatment resulted in obvious and persistent changes of EMT marker proteins in these cells, including the reduction of $\mathrm{CDH} 1$, and the increase of $\mathrm{CDH} 2$ and Vimentin (Fig. 1a,b, and western blot quantification in Supplementary Fig. 12). Under these circumstances, the SMAD7-mediated negative feedback loop of TGF $\beta$ signalling was disrupted in these cancer cell lines, leading to sustained responses of EMT.

miR-182 is induced by TGF $\beta$ and suppresses SMAD7 translation. The discrepant expression of SMAD7 transcript and protein indicated the presence of SMAD7 post-transcriptional regulation responding to TGF $\beta$. Since miRNA is a widely known mechanism for such regulation, we analysed whether miRNAs were responsible for the suppression of SMAD7 protein. Using the in silico miRNA target prediction software TargetScan ${ }^{21,22}$, we found several potential miRNA binding sites at the $3^{\prime}$ UTR of SMAD7 (Supplementary Table 1). Among these miRNAs, miR-182 was previously reported to be involved in cancer metastasis and TGF $\beta$ signalling by us and others ${ }^{19,23}$, and thus represented a promising candidate to interfere TGF $\beta$ regulation of SMAD7. We cloned SMAD7 $3^{\prime}$ UTR sequence containing the predicted miR-182 binding site for luciferase reporter assays. The luciferase activity of SMAD7 $3^{\prime}$ UTR was significantly reduced on miR-182 transfection in HeLa cells. Moreover, a 3-bp mutation in the seed sequence of the binding site abrogated the reduction of luciferase activity in response to miR-182 (Fig. 2a; Supplementary Fig. 1). In addition, treatment of the cells with an oligonucleotide inhibitor of miR-182 effectively restored the luciferase activity of SMAD7 $3^{\prime} \mathrm{UTR}$ (Fig. 2a). Therefore, SMAD7 $3^{\prime} \mathrm{UTR}$ is a direct target of miR-182.

To determine whether SMAD7 was regulated by miR-182 endogenously, we analysed miR-182 expression levels in the breast and lung cancer cell lines (Supplementary Fig. 2A) and assessed the effects of miR-182 overexpression or inhibition in these cells. miR-182 was stably overexpressed in SCP28, 4T1 and A549 (Supplementary Fig. 2B). Although SMAD7 mRNA was not obviously affected by miR-182 (Fig. 2b), a significant reduction of SMAD7 protein abundance was observed on miR-182 overexpression in these cancer cells (Fig. 2c). In addition, treating MCF10AT and EpRas with an anti-miR-182 oligonucleotide inhibitor ${ }^{23}$ elevated the protein level of SMAD7 but did not affect its mRNA level (Fig. 2b,c). We then used a sponge construct ${ }^{23}$ to 
a



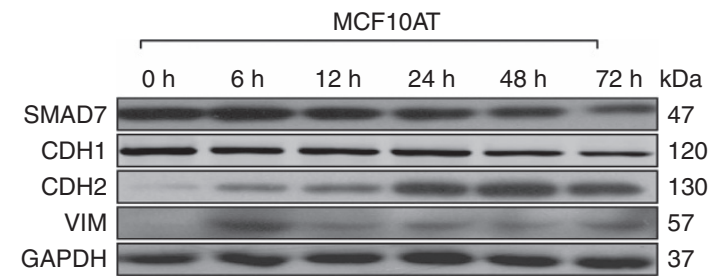


Figure 1 | TGF $\beta$ stimulation of cancer cells increases SMAD7 mRNA level but not the protein level. (a) SMAD7 mRNA expression (left), and western blot analyses of SMAD7 and EMT markers (right) after TGF $\beta$ stimulation for the indicated time duration in MCF10AT $(n=3)$. (b) SMAD7 mRNA expression (left), and western blot analyses of SMAD7 and EMT markers (right) in the indicated cell lines after $72 \mathrm{~h}$ of TGF $\beta$ treatment $(n=3) .{ }^{\star} P<0.05$, ${ }^{\star \star} P<0.01$, ${ }^{\star \star \star} P<0.001$ versus control by student's $t$-test. Error bars are defined as s.d.

a

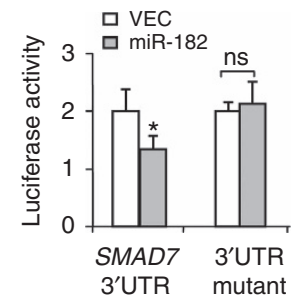

C

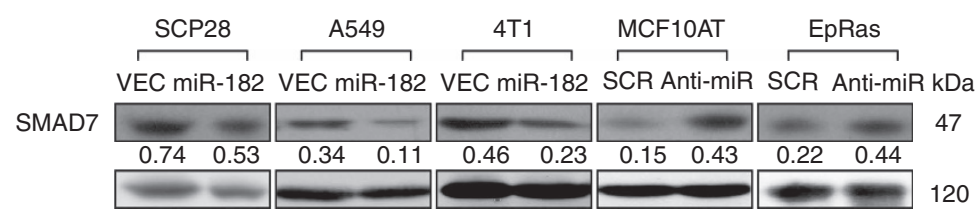

e


f b
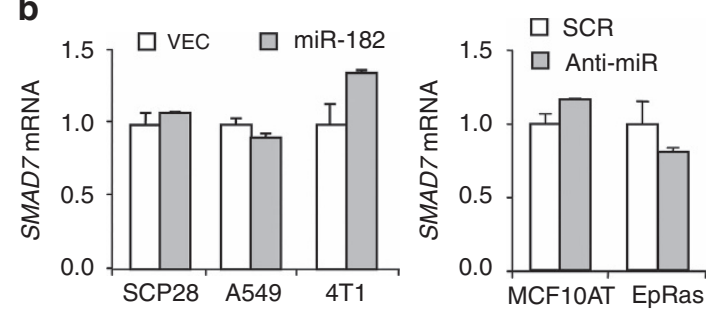

d


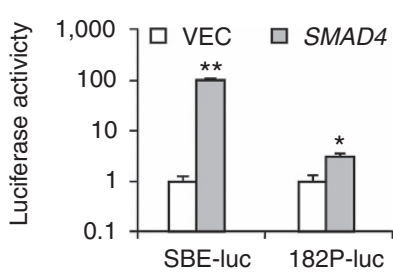

Figure 2 | miR-182 directly targets SMAD7 and is induced by TGFß. (a) Luciferase reporter assays of SMAD7 wild-type and mutated 3'UTR with miR-182 overexpression and inhibition $(n=4)$. (b) Endogenous SMAD7 mRNA levels after miR-182 overexpression and inhibition in the indicated cancer cells $(n=3)$. (c) SMAD7 protein levels after miR-182 overexpression and inhibition in the indicated cancer cells. Numbers denote western blot quantification of SMAD7 normalized to GAPDH. (d) miR-182 expression in SCP28 after TGF $\beta$ treatment for the indicated time duration or SMAD4 transfection ( $n=3$ ). (e) miR-182 expression after $48 \mathrm{~h}$ of TGF $\beta$ stimulation in the indicated cell lines $(n=3)$. (f) Luciferase reporter assays of miR-182 promoter in HeLa cells after TGF $\beta$ stimulation or SMAD4 overexpression $(n=4)$. The SBE reporter serves as a positive control. Anti-miR, miR-182 oligonucleotide inhibitor; VEC, empty vector control; SCR, scrambled siRNA control. ${ }^{\star} P<0.05,{ }^{\star \star} P<0.01, \mathrm{~ns}$, not significant by student's $t$-test. Error bars are defined as s.d.

inhibit miR-182 in the lung adenocarcinoma cell line NCI-H1299. The inhibition efficacy of the sponge was validated with $3^{\prime}$ UTR of a known miR-182 target gene MTSS1 (ref. 23) (Supplementary
Fig. 2C). It was found that the sponge also led to in SMAD7 protein elevation (Supplementary Fig. 2D). Therefore, miR-182 suppresses SMAD7 expression at its protein level. In addition, we 
found that miR-182 neither upregulated the E3 ligase of SMAD7, Arkadia $^{24}$, nor changed the ubiquitination status of SMAD7 protein (Supplementary Fig. 3). Taken together, these data indicate that miR-182 target SMAD7 for translational inhibition.

Previously it was reported that miR-182 expression could be induced by TGF $\beta$ in gallbladder cancer and glioma cells ${ }^{19,25}$. Altogether with the fact that miR-182 suppresses SMAD7 specifically at the protein level, this could explain the apparent disassociation of SMAD7 mRNA and protein expression following TGF $\beta$ treatment. Therefore, we assessed whether miR-182 could be induced by TGF $\beta$ in the above breast and lung cancer cell lines, and found that miR-182 was rapidly induced by TGF $\beta$ in SCP28. A nearly 5-fold increment of miR-182 transcript was observed $6 \mathrm{~h}$ after TGF $\beta$ treatment (Fig. 2d). miR-182 transcription was activated by the transfection of SMAD4 as well (Fig. 2d). The induction of miR-182 by TGF $\beta$ treatment was also confirmed in other breast and lung cancer cell lines (Fig. 2e). In silico analysis of the $2.5 \mathrm{~kb}$ promoter region of the miR-182 gene revealed several potential SBE sites. Therefore, we constructed the luciferase reporter of this region and found that it was responsive to both TGF $\beta$ treatment and SMAD4 overexpression (Fig. 2f). Thus, miR-182 is both a downstream target of TGF $\beta$ and a direct suppressor of SMAD7 in these cancer cell lines.

miR-182 promotes cancer cell migration and tumour invasion. The suppression of SMAD7 by miR-182 indicated that miR-182 might play a role in cancer cell EMT, migration and invasion. Therefore, we investigated the effects of miR-182 in these processes. Although we did not observe obvious morphological changes in cancer cells after miR-182 overexpression (Supplementary Fig. 4A), the EMT marker proteins, namely CDH1, $\mathrm{CDH} 2$ and Vimentin, were modestly regulated by miR-182. In A549 cells, the overexpression of miR-182 led to a slight reduction of $\mathrm{CDH} 1$ and induction of $\mathrm{CDH} 2$ and Vimentin. Reciprocally, miR-182 inhibition in EpRas by the oligonucleotide inhibitor resulted in CDH1 up-regulation, as well as CDH2 and Vimentin suppression (Fig. 3a). The sponge inhibitor also led to the suppression of $\mathrm{CDH} 2$ and Vimentin in NCI-H1299 (Fig. 3a).

Next, the effects of miR-182 in cancer cell migration and invasion were tested. Forced expression of miR-182 markedly enhanced the transwell invasiveness of SCP28 to a similar extent of TGF $\beta$ treatment (Fig. 3b). Meanwhile, miR-182 inhibition with a



b

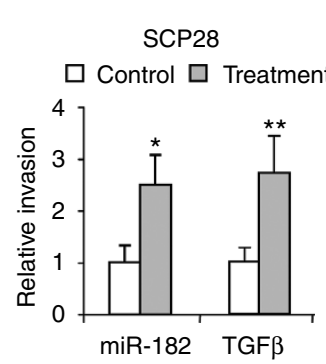

d

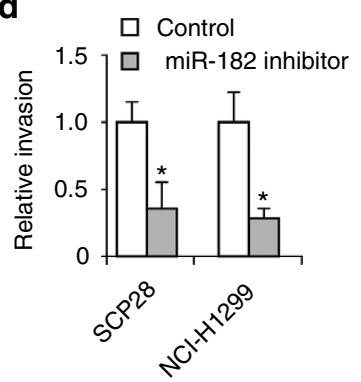

$\square \mathrm{SCR}+\mathrm{VEC}$

$\square$ Anti-miR + VEC

- Anti-miR + miR-182
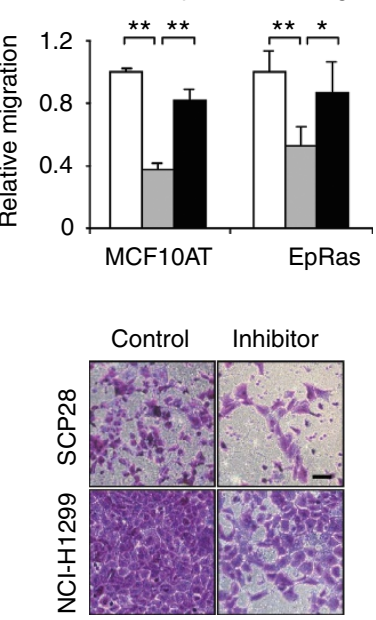

e



f



Tumour border Intratumour Tumour border Intratumour

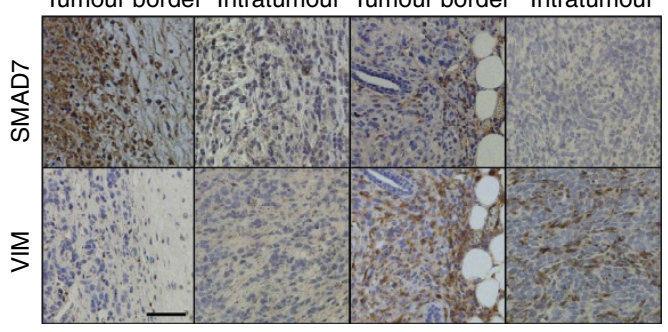

g
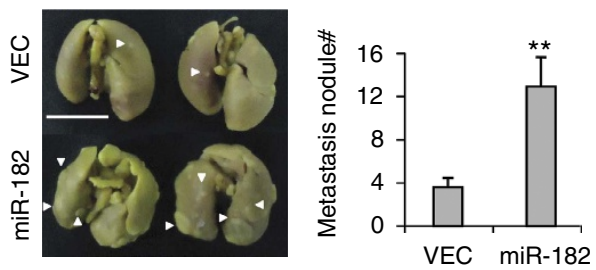

Figure 3 | The effects of miR-182 on cancer cell invasiveness and tumour metastasis. (a) Protein levels of EMT markers and SMAD7 in cancer cells after miR-182 overexpression or silencing. (b) Transwell invasion assays of SCP28 with miR-182 overexpression or TGF $\beta$ treatment $(n=4)$. (c) Migration assays of MCF10AT and EpRas cells with miR-182 silencing and exogenous miR-182 overexpression $(n=4)$. (d) Transwell invasion assays of cancer cells treated with miR-182 inhibitors. SCP28 was treated with the oligonucleotide inhibitor and $\mathrm{NCl}-\mathrm{H} 1299$ with the sponge inhibitor $(n=4)$. (e) Tumour edge $\mathrm{H} \& \mathrm{E}$ staining of 4T1 cells with miR-182 overexpression. (f) SMAD7 and Vimentin IHC staining of 4T1 primary tumours. (g) Whole-lung images and quantification of metastasis nodules at the lung surface on day 35 after orthotopic injection of 4 T1 cells $(n=10$ mice per group). Arrowheads point to metastasis nodules. Scale bars, $1 \mathrm{~cm}(\mathbf{g})$ and $150 \mu \mathrm{m}(\mathbf{d}-\mathbf{f}) .{ }^{\star} P<0.05,{ }^{\star \star} P<0.01$, ns, not significant by student's $t$-test. Error bars are defined as s.d. 
the oligonucleotide or the sponge inhibitor significantly impaired the migration and invasion of MCF10AT, EpRas, SCP28 and NCI-H1299 cells (Fig. 3c,d). In addition, re-introducing miR-182 into the cells by overexpression rescued the impaired cell migration caused by miR-182 inhibition (Fig. 3c). Taken together, these data demonstrate that though miR-182 has a weaker effect on EMT regulation, it mimics TGF $\beta$ signalling to promote cancer cell migration and invasion.

To further assess the function of miR-182 in tumour invasion and metastasis in vivo, we used the orthotopic transplantation model of $4 \mathrm{~T} 1$ breast cancer cells. 4T1 cells with stable miR-182 overexpression and the control cells were implanted into the mammary fat pads of BALB/c mice. No difference in the rate of primary tumour growth was detected (Supplementary Fig. 4B). However, when the tumours were histologically examined, we observed markedly enhanced invasiveness of the miR-182expressing tumours. These tumours had widely-spread invasive fronts characterized by infiltrated tumour-stroma interface and the presence of tumour cells intermingled in the stromal side. In contrast, the control tumours were well contained with intact tumour-stroma interface (Fig. 3e). The augmented invasiveness was accompanied by attenuated SMAD7 and enhanced Vimentin both at the edge and the interior areas of the tumours, as revealed by immunohistochemistry (IHC) analyses (Fig. 3f). In addition, when the mice were euthanized and their lungs were examined at the 5th week post tumour transplantation, significantly more metastases were observed in the overexpression group. The number of metastasis nodules on the pulmonary surface was over 3 -fold higher than the control (Fig. 3g). These findings demonstrated a functional role of miR-182 to promote tumour invasion and spreading in vivo.

miR-182 is required for TGF $\beta$-induced EMT and invasion. Since miR-182 suppressed TGF $\beta$-induced upregulation of SMAD7, we reasoned that this miRNA could be critical for cancer cell responses to TGF $\beta$. Therefore, we treated MCF10AT and EpRas with the anti-miR-182 oligonucleotide together with TGF $\beta$, and analysed the cellular responses in EMT. Western blot and immunofluorescence analyses showed that miR-182 inhibition resulted in upregulation of SMAD7 protein, and suppressed the changes of EMT markers in response to TGF $\beta$ in the cancer cells (Fig. 4a,b). Without miR-182 inhibition, TGF $\beta$ treatment markedly reduced the epithelial marker $\mathrm{CDH} 1$ that was localized to the cell membrane, while the mesenchymal markers $\mathrm{CDH} 2$ and Vimentin were significantly elevated (Fig. 4b). However, these TGF $\beta$-induced molecular events were completely abolished when the cells were simultaneously treated by the miR-182 inhibitor (Fig. 4b). Such changes in the expression of EMT markers, as well as EMT-related transcription factors such as a

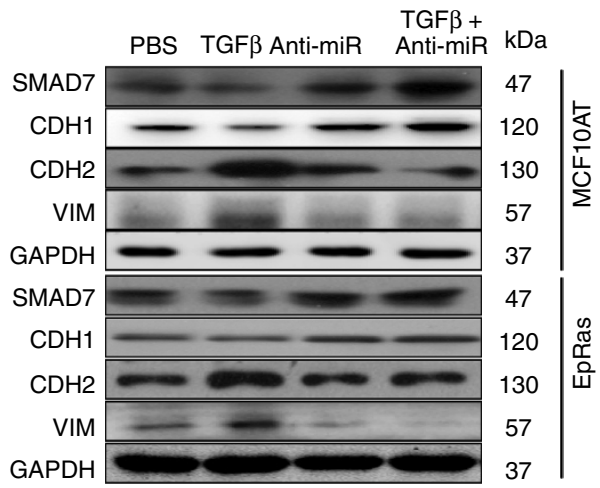

c



d

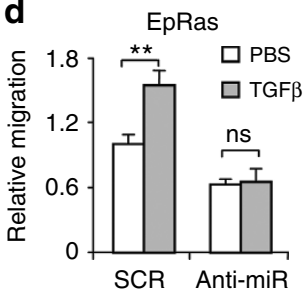

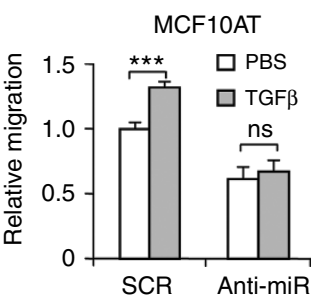

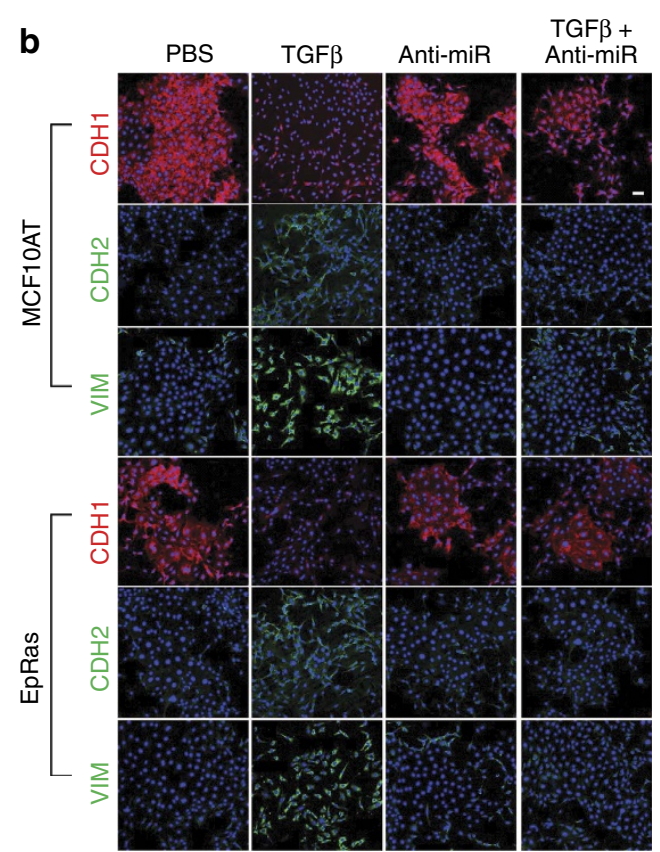

$\mathbf{e}$

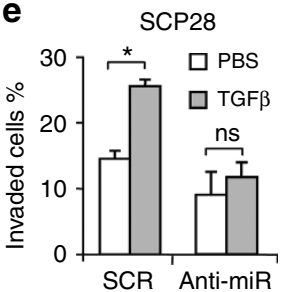

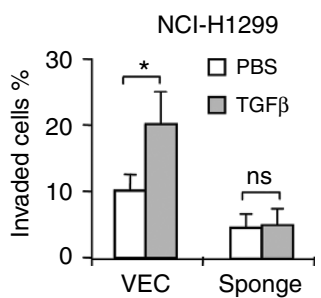

Figure 4 | miR-182 silencing impairs TGF $\beta$-induced EMT and invasion. (a) Protein expression of EMT markers and SMAD7 in EpRas and MCF10AT cells after TGF $\beta$ stimulation and miR-182 inhibition. (b) Immunofluorescence analyses of EMT makers in EpRas and MCF10AT. (c) Morphological changes of EpRas and MCF10AT cells. (d) Migration of EpRas and MCF10AT cells $(n=4)$. (e) Transwell invasion of SCP28 and NCI-H1299 cells $(n=4)$. Scale bars, $50 \mu \mathrm{m}$. ${ }^{\star} P<0.05,{ }^{\star \star} P<0.01,{ }^{\star \star \star} P<0.001$ by student's $t$-test. Error bars are defined as s.d. 
a

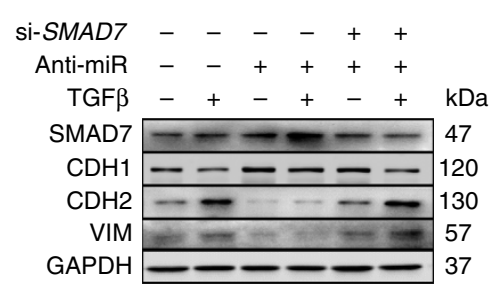

d

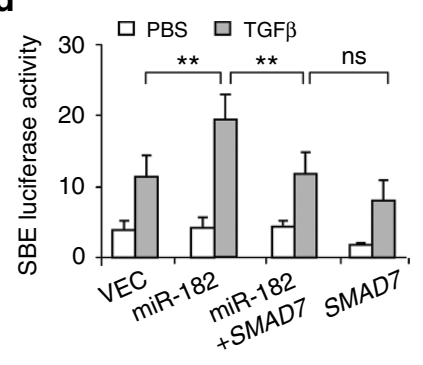

b

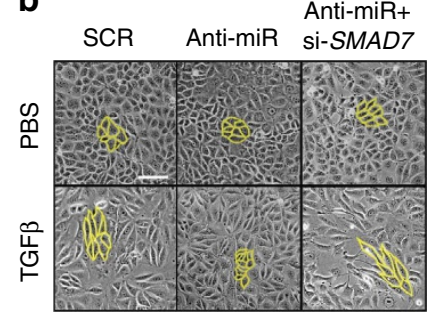

e

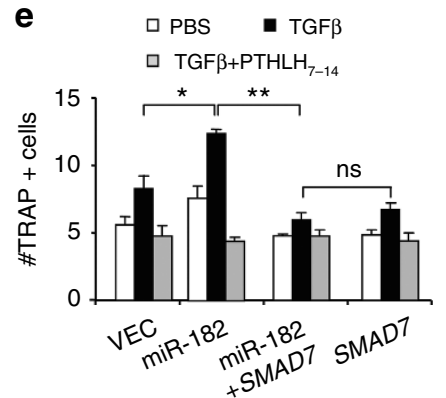

C

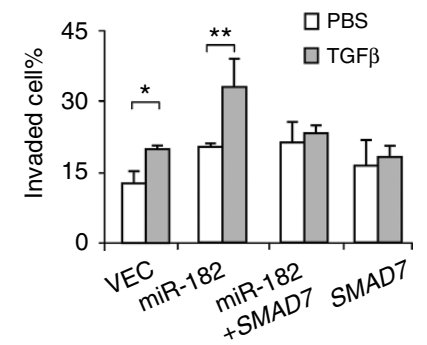

f

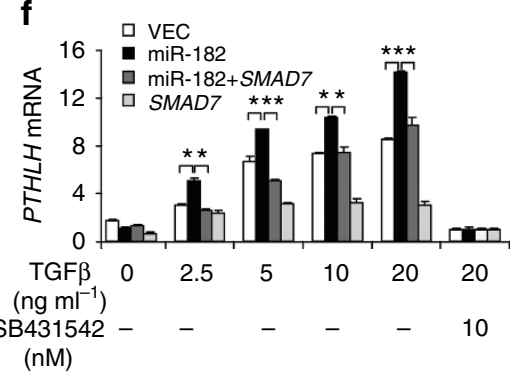

Figure 5 | SMAD7 rescued the effect of miR-182 in TGF $\beta$-induced EMT and osteoclastogenesis. (a) Protein levels of EMT markers and SMAD7 in EpRas with TGF $\beta$ stimulation, miR-182 inhibition and SMAD7 silencing. (b) EMT morphological changes of EpRas with TGF $\beta$ stimulation, miR-182 inhibition and SMAD7 silencing. (c) Transwell invasion of SCP28 with TGF $\beta$ stimulation, miR-182 and SMAD7 overexpression ( $n=4)$. (d) SBE luciferase reporter assays of HeLa cells with TGF $\beta$ stimulation, miR-182 and SMAD7 overexpression $(n=4)$. (e) Osteoclastogenesis of primary bone marrow in conditioned medium of SCP28 with miR-182 and SMAD7 overexpression. Tumour cells were treated with TGF $\beta$, followed by conditioned medium harvest and primary bone marrow culture. The peptide antagonist PTHLH $7-34(0.5 \mu \mathrm{M})$ was added into the bone marrow culture $(n=4)$. (f) PTHLH expression in SCP28 treated with TGF $\beta$ at different concentration, and the TGF $\beta$ inhibitor SB431542 $(n=3)$. Scale bars, $100 \mu \mathrm{m}$. ${ }^{\star} P<0.05$, ${ }^{\star \star} P<0.01$ by student's $t$-test. Error bars are defined as s.d.

TWIST1 and ZEB1, were also observed by western blot and mRNA qPCR analyses in these cells (Fig. 4a, Supplementary Figs 5 and 6). Notably, miR-182 inhibition also quenched the morphological shift of cancer cells induced by TGF $\beta$. TGF $\beta$ treatment led the cancer cells transform from the cobble stonelike appearance to the elongated, spindle-like mesenchymal shape. In contrast, no manifest changes were observed in cell morphology after the treatment of TGF $\beta$ along with the inhibitor (Fig. 4c).

In addition to the observations of molecular and morphological changes, miR-182 silencing effectively suppressed cell migration and invasion caused by TGF $\beta$ in EpRas, MCF10AT, SCP28 and NCI-H1299. In the presence of miR-182 inhibitors, TGF $\beta$ failed to enhance the motility or invasiveness of cancer cells (Fig. $4 \mathrm{~d}, \mathrm{e}$ ). Thus, these data suggest that miR-182 is critical for TGF $\beta$ responses of cancer cells in EMT and invasion.

As a comparison, we also analysed the role of miR-182 in TGF $\beta$ responses of non-malignant mammary epithelial cells MCF10A and HMLE. Unlike in cancer cells, TGF $\beta$ treatment of these normal cells failed to induce the expression of miR-182, and thus an obvious increase of SMAD7 protein was observed following TGF $\beta$ stimulation (Supplementary Fig. 7A,B). Concordantly, miR-182 did not obviously alter the TGF $\beta$ responsiveness of the SBE reporter, or TGF $\beta$-induced cell apoptosis in MCF10A (Supplementary Fig. 7C,D). Therefore, the positive feedback loop of TGF $\beta$-miR-182 seems to be specific to cancer cells.

miR-182 promotes EMT and invasion by suppressing SMAD7. To corroborate that SMAD7 suppression mediates the role of miR-182 in cancer cell TGF $\beta$ responses, we treated EpRas cells with a SMAD7 siRNA inhibitor (Supplementary Fig. 8A) in addition to TGF $\beta$ stimulation and miR-182 inhibition. While miR-182 suppression led to SMAD7 induction on TGF $\beta$ treatment and abolished TGF $\beta$-induced EMT phenotypes, simultaneous treatment with the SMAD7 inhibitor restored cancer cell responses to TGF $\beta$. The SMAD7 protein level was effectively suppressed, along with the manifest changes of EMT markers and cellular morphology after TGF $\beta$ stimulation, despite the inhibition of miR-182 (Fig. 5a,b). The assays were repeated in MCF10AT cells as well, and the same changes in EMT markers were observed (Supplementary Fig. 8B), suggesting that SMAD7 induction mediates the effects of miR-182 inhibition in cancer cell EMT.

Reciprocally, we stably overexpressed both SMAD7 and miR-182 in SCP28 (Supplementary Fig. 8C), followed by TGF $\beta$ treatment. As expected, SMAD7 overexpression abolished the effects of miR182 in cancer cell invasion. With forced expression of SMAD7, miR-182 was no longer able to boost the cancer cell invasion after TGF $\beta$ stimulation (Fig. 5c). Similarly, treating the cells with the TGF $\beta$ receptor inhibitor SB431542 also abolished the effect of miR-182 on cell invasion (Supplementary Fig. 8D). The same phenomenon was observed in $4 \mathrm{~T} 1$ cells when miR-182 and SMAD7 were both overexpressed, following assessments of cell migration and invasion (Supplementary Fig. 8E). We also analysed the TGF $\beta$ response of SCP28 cells with SBE luciferase reporter assays. TGF $\beta$ treatment induced the SBE reporter activity, and miR-182 overexpression further enhanced the response. In contrast, SMAD7 diminished the enhancing effect of miR-182 on SBE activity, although the reporter was still partially responsive to TGF $\beta$ stimulation. Importantly, in the presence of SMAD7 overexpression, miR-182 failed to enhance the SBE response to TGF $\beta$ (Fig. $5 \mathrm{~d}$ ). These data showed that miR-182 promotes cancer cell responses to TGF $\beta$ by suppressing SMAD7. 
miR-182 promotes osteoclastogenesis and bone metastasis. In addition to induction of EMT and cancer cell invasion, TGF $\beta$ signalling also underlies the process of skeleton microenvironment remodelling and osteoclast differentiation induced by cancer cells for metastasis to bone, a physiological reservoir of TGF $\beta$. Therefore, we further analysed the role of miR-182 in TGF $\beta$ signalling during bone metastasis. We first assessed the ability of cancer cells to induce the differentiation of pre-osteoclasts in murine bone marrow. Primary marrow was cultured in the conditioned medium from SCP28 cells with miR-182 and/or SMAD7 overexpression and osteoclast maturation was assessed by tartrate-resistant acid phosphatase (TRAP) staining. As expected, TGF $\beta$ treatment of SCP2 8 before conditioned medium harvest promoted osteoclast maturation when bone marrow was cultured in the conditioned medium (Fig. 5e; Supplementary Fig. 9A). In contrast, adding TGF $\beta$ directly into the bone marrow culture produced no difference of osteoclastogenesis (Supplementary Fig. 9B). Notably, miR-182 overexpression was able to augment cancer cell response to TGF $\beta$ for osteoclast induction. However, SMAD7 expression completely blocked the effect of miR-182 in osteoclast maturation after TGF $\beta$ treatment (Fig. 5e). Previously we and others have showed that cancer cells, in response to TGF $\beta$, express and secret PTHLH, which stimulates RANKL expression of osteoblasts and induces osteoclast maturation ${ }^{9,26-28}$. Concordantly, miR-182 overexpression in SCP28 enhanced PTHLH expression and secretion of SCP28 following TGF $\beta$ treatment, as well as Rankl expression of the pre-osteoblast $\mathrm{C} 2 \mathrm{C} 12$ cells when cultured in SCP28 conditioned medium, while SMAD7 or the TGF $\beta$ inhibitor SB431542 abrogated such effects of miR-182 (Fig. 5f, Supplementary Fig. 10A,B). In addition, other TGF $\beta$ target genes, such as IL11 (ref. 10), were enhanced by miR-182 and suppressed by SMAD7 in SCP28 as well. In contrast, CSF1, which was also involved in osteoclastogenesis $^{10}$ but was not responsive to TGF $\beta$, was not regulated by miR-182 either (Supplementary Fig. 10C). These observations corroborated that miR-182 enhances TGF $\beta$ signalling of cancer cells. We also found that the peptide inhibitor of PTHLH, PTHLH ${ }_{7-14}$ (ref. 9), could completely block the osteoclastogenesis effect of miR-182 (Fig. 5e), indicating that PTHLH is the main downstream mediator of miR-182 to regulate TGF $\beta$-induced osteoclast maturation.

Then, we analysed the consequences of miR-182 and SMAD7 overexpression in breast cancer bone metastasis in vivo. The miR182-overexpressing or control SCP28 cells were intracardially injected into the circulation of nude mice followed by bioluminescent imaging of bone metastasis. First we confirmed that miR-182 was still overexpressed in the metastasis tumours caused by miR-182-overexpressing cancer cells (Supplementary Fig. 11A). miR-182 overexpression significantly accelerated the outgrowth of cancer cells in bone, and caused more severe bone damages (Fig. 6a-c). Interestingly, SMAD7 reversed the phenotypes and dampened bone metastasis. More importantly, with forced expression of SMAD7, miR-182 caused no obvious difference in the formation of bone metastases (Fig. 6a-c). Concordant to the difference in bone damages, there were significantly more osteoclasts along the tumour-bone interface when the tumour cells overexpressed miR-182, and this increment was suppressed by SMAD7 overexpression (Fig. 6d,e). Additionally, miR-182 and SMAD7 led to opposite changes in the level of SMAD3 phosphorylation and PTHLH expression in bone metastases (Fig. 6d; Supplementary Fig. 11B).

To further validate the role of miR-182 in bone metastasis of cancer cells, SCP28 cells with concurrent overexpression of miR-182 and its target genes were injected intratibially into the bone marrow of mice, followed by analyses of tumour growth and bone damage. Again, we found that miR-182 accelerated the growth of cancer cells in bone and osteolytic damage of the skeleton. SMAD7 overexpression completely blocked such effects of miR-182. In contrast, MTSS1, the other miR-182 target gene that also mediates the pro-invasive function of miR-182 (ref. 23), had no effects on SCP28 outgrowth in bone (Fig. 6f,g, Supplementary Fig. 11C). Taken together, these data suggest that miR-182 upregulates cancer cell response to microenvironmental TGF $\beta$ and bone metastasis by suppressing SMAD7.

miR-182 inversely correlates with SMAD7 in clinical samples. We analysed the expression of miR-182 in clinical breast tumour samples of The Cancer Genome Atlas (TCGA) database and found that miR-182 expression was significantly higher in breast tumours than in normal tissues. The difference was still significant when the analyses were performed for individual breast cancer molecular subtypes (Fig. 7a). A direct comparison of paired tumour and normal tissues of the same patients also revealed upregulation of miR-182 in most of the cases (Fig. 7b). The correlation of miR-182 expression with clinical metastasis was also analysed for the TCGA cohort, and it was found that miR-182 elevation was linked to accelerated metastasis in triple negative breast cancer, a subtype characterized with poor prognosis (Fig. 7c). To further investigate the clinical relevance of SMAD7 suppression by miR-182, we examined a cohort $(n=24)$ of breast cancer clinical samples collected from Qilu Hospital by q-PCR of miR-182 and IHC staining of SMAD7. qPCR analysis of miR-182 expression in these tumour samples were first validated by in situ hybridization analysis with a miR-182 probe (Fig. 7d). Although we did not observe obvious differences of SMAD7 mRNA levels in the tumours expressing high or low levels of miR-182 (Fig. 7e), the SMAD7 protein level was significantly lower in the samples with abundant miR-182. Only 1 out of the 12 samples with high levels of miR-182 showed strong staining of SMAD7, as compared with 7 of the 12 samples with low levels of miR-182 ( $P=0.027$, Fig. $7 \mathrm{f})$, corroborating an inverse correlation between SMAD7 protein and miR-182. The linkage of miR-182 expression with the SMAD7 protein, but not with the SMAD7 mRNA, was concordant to our observation that miR-182 targeted SMAD7 to suppress its protein level (Fig. 2b,c). Collectively, our data support the conclusion that miR-182 targets SMAD7 to enhance TGF $\beta$ signalling in cancer cell lines and human tumour samples.

\section{Discussion}

Since the identification of SMAD7 as a negative regulator of TGF $\beta$ (ref. 29), it has been assumed to serve as a brake of TGF $\beta$ signal transduction. Indeed, the SMAD7 mRNA can be rapidly induced up to 4-10 folds by TGF $\beta$ treatment in numerous types of cells ${ }^{30,31}$; meanwhile, upregulation of SMAD7 to the same magnitude in mucosal mononuclear cells of inflammatory bowel disease patients as compared with the normal controls was sufficient to desensitize the cells to TGF $\beta$ (ref. 32). Forced expression of SMAD7 in cancer cells also blocked the cellular responses to TGF $\beta$ (ref. 33). These observations support a role of TGF $\beta$-induced SMAD7 to limit the propagation of TGF $\beta$ signalling. However, it is also well known that TGF $\beta$ treatment of cancer cells usually instigates a seemingly unchecked responses, including altered gene expression, EMT, invasiveness and metastasis microenvironment remodelling ${ }^{1,34}$. These manifest and persistent changes raise the question regarding whether and how cancer cells can avert the turnoff signal from I-SMADs. Although it has been well established that SMAD7 mRNA can be activated by TGF $\beta$ (refs 16,29,30,35-37), only very few studies reported the elevation of SMAD7 protein following TGF $\beta$ stimulation in cancer cells ${ }^{38}$. In addition, most of the functional 
a
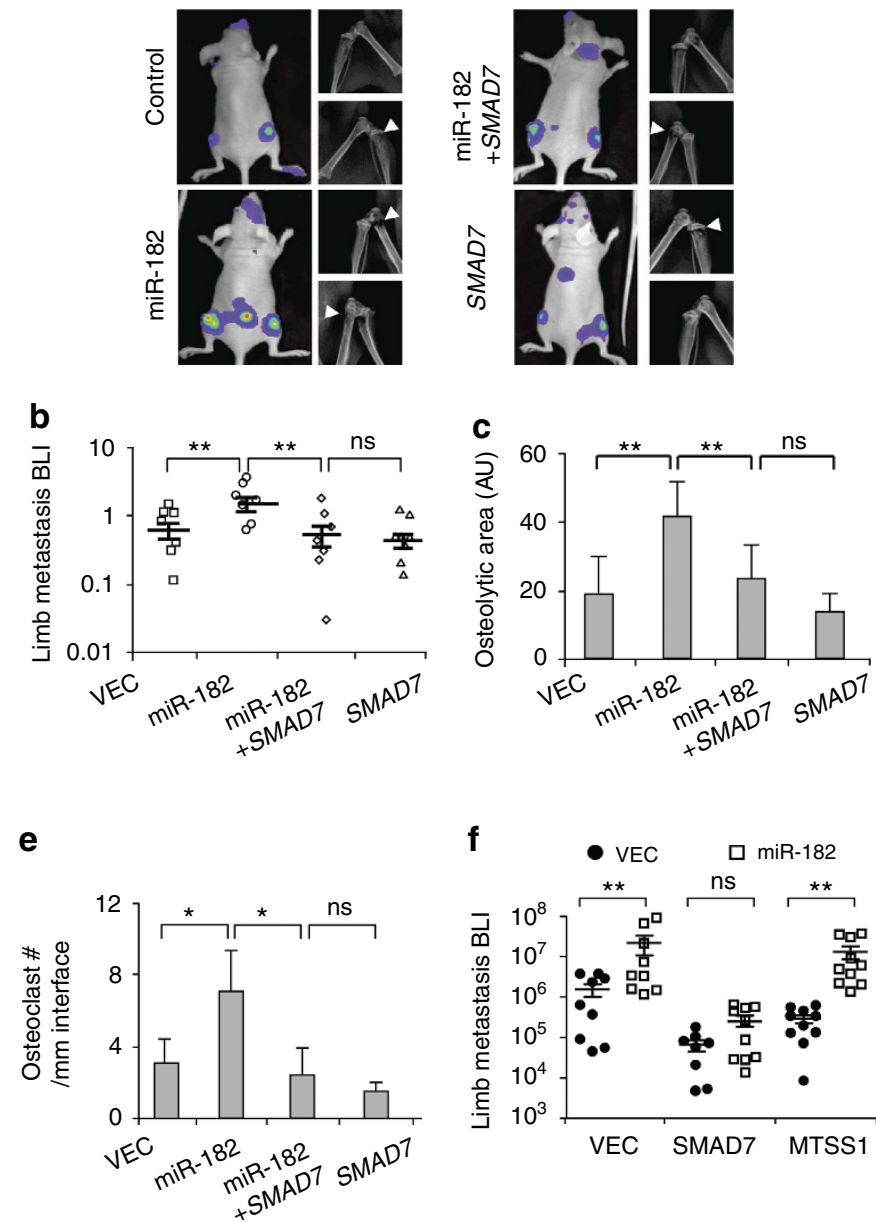

d

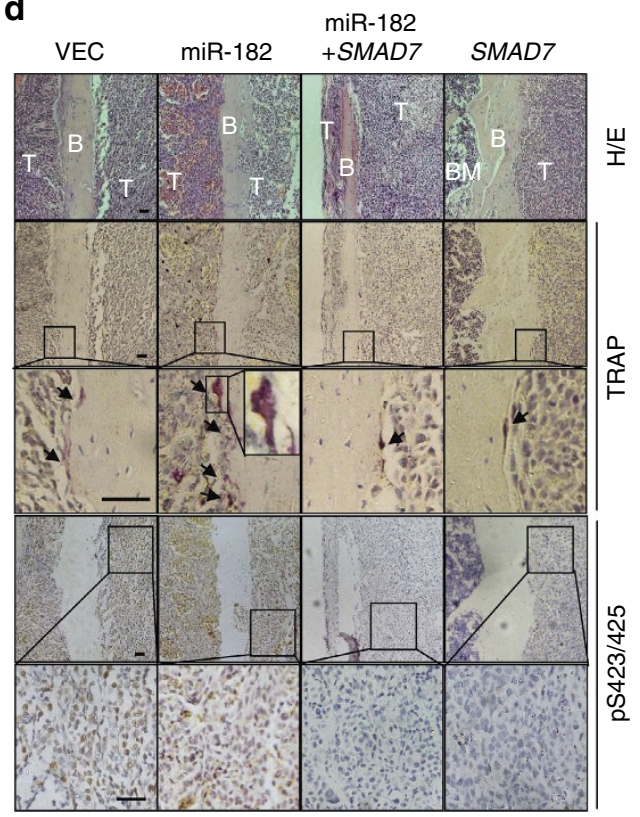

g


Figure 6 | The role of miR-182 in bone metastasis. (a) Bioluminescent imaging (BLI) and X-ray of bone metastases by SCP28 cells. Arrowheads denote areas of overt osteolysis. (b) BLI quantitation of limb metastasis by SCP28 4 weeks after intracardiac injection $(n=7)$. (c) Quantitation of osteolytic areas caused by SCP28 $(n=7)$. (d) Representative H\&E images, osteoclast TRAP staining, and IHC analyses of SMAD3 phosphorylation of bone metastases by SCP28. Arrows point to TRAP + osteoclasts along the tumour-bone interface. Scale bars, $50 \mu \mathrm{m}$. (e) Osteoclast numbers along the tumour-bone interface $(n=7)$. (f) BLI quantitation of limb metastasis by SCP28 4 weeks after intratibial injection $(n=10)$. (g) Quantitation of osteolytic area sizes caused by SCP28 cells after intratibial injection $(n=10)$. AU, arbitrary unit. ${ }^{\star} P<0.05,{ }^{\star \star} P<0.01$ by student's $t$-test. Error bars are defined as s.d.

studies of SMAD7 that validated its TGF $\beta$-inhibitory role in cancer have been carried out with ectopic overexpression of the gene ${ }^{33,39-42}$, thus not necessarily reflecting what actually occurs in cancer cells after TGF $\beta$ treatment. In this study we observed an unexpected phenomenon in a panel of breast and lung cancer cell lines that in spite of SMAD7 mRNA induction in immediate response to TGF $\beta$, the protein abundance of SMAD7 was not elevated, or even slightly reduced following the stimulation (Fig. 1c), indicating the inefficiency of SMAD7-mediated feedback to restrain TGF $\beta$ signalling in these cancer cells and in the studied context. We further found that these cancer cells achieved this via TGF $\beta$-induced expression of miR-182, which suppressed SMAD7 and thus endorsed the extended activation of TGF $\beta$ signalling leading to EMT and metastasis. Concordantly, miR-182 silencing with oligonucleotide or sponge inhibitors reactivated the self-limiting signal of the TGF $\beta$ pathway and impaired cancer cell responses in morphological transformation and microenvironment remodelling. Thus our study reveals a route of cancer cells to circumvent the abnegation of TGF $\beta$ signalling, and provides an explanation of sustained responses of cancer cells to TGF $\beta$ stimulation. A remaining question is whether this route is specific to the studied cell lines or other cancer cells could also adopt the same scheme to enhance TGF $\beta$ responses.

Balance of positive and negative inputs is crucial to maintain the proper functions of signal transduction. However, cancer cells are often characterized by the loss of control of signal pathways that govern cell proliferation and malignant progression ${ }^{43}$. The miR-182-mediated disruption of TGF $\beta$ negative feedback provides a good example of how central pathways can be disregulated in malignant cells. Rationally, another way to break the loop holding back TGF $\beta$ signalling would be genetic mutations of SMAD7, which have been reported in cancers ${ }^{44,45}$. However, missense and nonsense SMAD7 mutations have been mainly observed in colorectal cancer and are not linked to increased tumour metastasis ${ }^{45-47}$. Thus our findings reveal a novel mechanism for cancer cells to enhance TGF $\beta$ signalling and promote metastasis. It remains to be further studied what happens in normal cells, although our preliminary results showed that in non-cancerous mammary epithelial cells MCF10A and HMLE TGF $\beta$ treatment did not result in miR-182 elevation and thus the SMAD7 protein was induced (Supplementary Fig. 7). Therefore, the positive feedback loop of TGF $\beta$ signalling and miR-182 expression seems to be disconnected in 
a

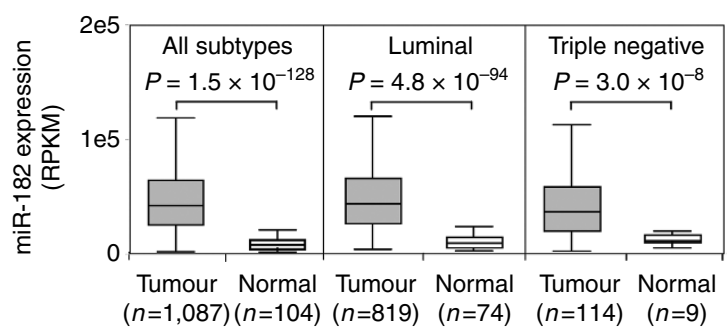

C

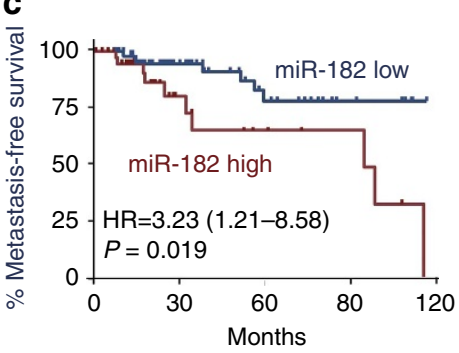

b

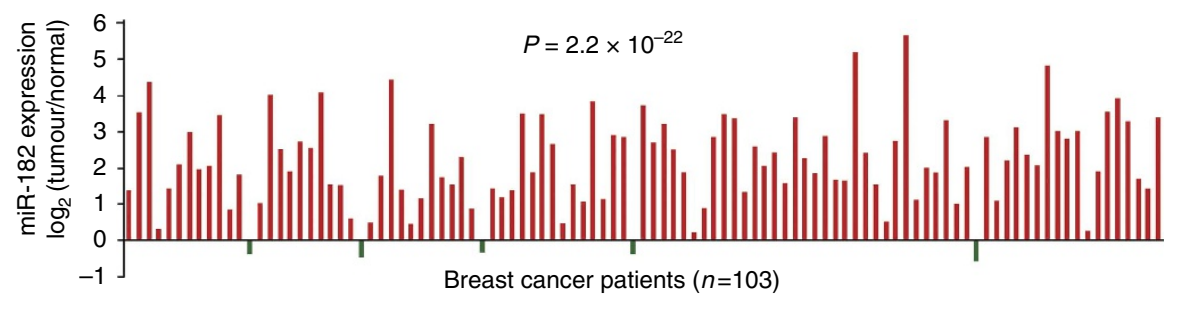

d

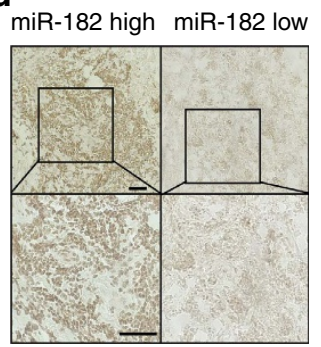

e

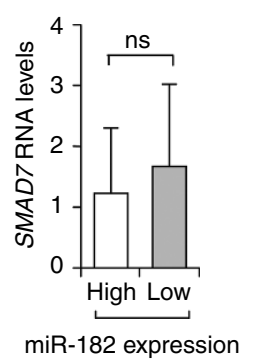

$\mathbf{f}$



Figure 7 | miR-182 was upregulated and negatively correlated with SMAD7 protein in clinical breast tumours. (a) Comparison of miR-182 expression in TCGA breast invasive carcinoma and normal tissues. (b) Pair-wise comparison of TCGA breast invasive carcinoma and normal tissues of the same patients. Log-ratios of miR-182 expression were shown. (c) Metastasis-free survival of the TCGA triple negative breast cancer patients with high or low levels of miR-182 expression. (d) miR-182 in situ hybridization of the Qilu cohort samples with different miR-182 levels. (e) qPCR analyses of SMAD7 mRNA in the Qilu cohort of human breast tumour samples $(n=24)$ with different miR-182 levels. (f) SMAD7 protein levels in Qilu samples with different miR-182 levels. Representative IHC images were shown on the right. Scale bars, $50 \mu \mathrm{m} .{ }^{\star} P<0.05$, ns, not significant by student's $t$-test. Error bars are defined as s.d.

normal epithelial cells, although the mechanism of insensitivity of miR-182 expression to TGF $\beta$ treatment in these cells is unclear.

As SMAD7 is a critical component in the TGF $\beta$ pathway, understanding how it is regulated is crucial to elucidate the role and mechanism of TGF $\beta$ signalling. While SMAD7 is known to be transcriptionally regulated by TGF $\beta$ and $\mathrm{NF \kappa B}$ pathways ${ }^{29,48,49}$, recent studies have reported that post-transcriptional regulation, such as protein acetylation and ubiquitination, plays key roles to control its protein level ${ }^{50,51}$. Accumulating evidence has also shown the involvement of miRNAs, including miR-21, miR-106-25 and miR-216a/217, in the regulation of SMAD7 (refs 52-54). In this study, we showed that miR-182 also targets SMAD7 to elevate cancer cell responses to TGF $\beta$ during metastasis. Notably, miR-182 selectively inhibits the protein level of SMAD7, but not its transcript level, which was confirmed by our expression analysis of clinical samples. In addition, the lack of correlation between I-SMAD mRNA and protein levels was recently noticed in pancreatic cancer ${ }^{55}$. Although multiple IHC analyses of SMAD7 have firmly established the correlation of SMAD7 protein expression with better prognosis of cancer patients ${ }^{56-58}$, the SMAD7 mRNA levels did not show such clinical relevance ${ }^{59,60}$. Collectively, these studies underscore the importance of post-transcriptional regulation of SMAD7 to coordinate TGF $\beta$ signalling in developmental and pathological conditions.
miR-182 is a pleiotropic miRNA that regulates circadian rhythm, immune system and DNA repair ${ }^{61-64}$. Its role in cancer has also been reported ${ }^{19,23,65-69}$. Although the majority of these studies demonstrated its pro-metastatic role in melanoma, glioma, ovarian cancer and breast cancer ${ }^{19,23,65-67}$, two recent studies indicated an opposite function of miR-182 in cancer cell migration and invasion 68,69 , suggesting the contextual dependence of its function in cancer metastasis. In this study we, for the first time, report a role of miR-182 in targeting SMAD7 to enhance TGF $\beta$ signalling and metastasis. The analyses were performed in multiple cell lines of breast and lung cancers, thus corroborating the findings of earlier reports ${ }^{19,23,65-67}$. Noticeably, our data showed that although miR-182 was required for TGF $\beta$-induced EMT, its overexpression only led to modest changes in the expression of EMT markers (Fig. 3a), and the regulation in cancer cell morphology was not obvious (Supplementary Fig. 4A). The role of miR-182 in osteoclast induction was also observed only with the supplement of TGF $\beta$ in vitro, or in bone where TGF $\beta$ is largely available. Therefore, miR-182 was a much less potent inducer of EMT than TGF $\beta$ itself, suggesting that SMAD7 suppression alone was not sufficient for fully activation of TGF $\beta$ signalling. In addition, it is also noticed that $S M A D 7$ overexpression failed to completely block cancer cell TGF $\beta$ responses as revealed by SBE activity, osteoclast differentiation and PTHLH induction (Fig. 5d-f), implying a SMAD7-independent mechanism for the oncogenic 
role of miR-182 and the TGF $\beta$ response of cancer cells. For example, the promotion of cell invasiveness by miR-182 might result from the synergistic action of SMAD7 and other downstream targets, such as MTSS1 (ref. 23).

miR-182 not only regulates TGF $\beta$ signalling by targeting SMAD7, but also is responsive to TGF $\beta$ signalling, thus constituting a selfenhancing circuit of the pathway. The TGF $\beta$ negative feedback loop mediated by I-SMADs has been well studied, and now our study show that this loop is antagonized by the miRNA-mediated positive feedback signal, which expands the spectrum of TGF $\beta$ regulatory network and further reveals the complexity of this pathway in cancer progression. In addition, our data demonstrated the aberrant up-regulation of miR-182 and its negative correlation with SMAD7 protein in tumours samples. Previously we also showed that miR182 expression was linked to elevated risk of metastasis in breast cancer patients ${ }^{23}$. Overall, our results demonstrate that miR-182 is a critical component of TGF $\beta$ signalling. The uncovering of this TGF $\beta$-miR-182 circuit will extend our comprehension of TGF $\beta$ network complexity and argue for miR-182 as a new option to target TGF $\beta$ signalling for cancer intervention.

\section{Methods}

Plasmid and cell lines. Overexpression of miR-182 and MTSS1 was performed in the pMSCV retroviral plasmid ${ }^{23}$. The miR-182 sponge plasmid was constructed by inserting eight tandemly arrayed miR-182-binding sites (50-AGTGTGAGTTC TAGGGTTTGCCAAA-30) into the $3^{\prime} \mathrm{UTR}$ of $\mathrm{dsRed}^{23}$. For $3^{\prime} \mathrm{UTR}$ reporter assays, a $0.8 \mathrm{~kb}$ fragment of $S M A D 73^{\prime}$ UTR was cloned into pMIR-REPORT (Ambion) with SpeI and SacI digestion. The miR-182 seed sequence was further mutated to $5^{\prime}$-ATGGGTAAT- $3^{\prime}$. The $2.1 \mathrm{~kb}$ fragment of MTSS1 $3^{\prime}$ UTR was cloned into pMIRReport $^{23}$. For promoter reporter assays, a $2.5 \mathrm{~kb}$ fragment upstream of the miR-182 gene was cloned into pGL3basic (Ambion) with NheI and XhoI digestion. The CDNA vector of human SMAD7 was provided by Dr Heldin ${ }^{29}$ and was subcloned to the pMSCV-hygro vector. All constructs were confirmed by sequencing. The plasmids were transiently transfected to target cells with lipofectamin 2000 (Invitrogen, cat.11668). To generate stable lines of overexpression or sponge knockdown, the plasmids were packaged into retrovirus with the amphotropic Phoenix packaging cell line and infected into target cells, followed by puromycin/hygromycin selection of the infected cells. MCF10A and MCF10AT and 4T1 cell lines were obtained from Dr Miller ${ }^{70}$. EpRas was obtained from Dr Weinberg ${ }^{8}$, SCP28 from Dr Massague ${ }^{20}$. NCI-H1299 (TCHu160) and A549 (TCHu150) were purchased from the Cell Bank of Type Culture Collection of Chinese Academy of Sciences. All the cell lines were confirmed as mycoplasma free by mycoplasma PCR tests.

Reagents. The mouse monoclonal SMAD7 antibody (R\&D, cat. MAB2029), mouse VIM antibody (BD Biosciences, cat. 550513) and mouse VIM antibody (Sigma, cat. V2258) were used for western blot and immunohistochemistry analyses. The mouse CDH2 antibody (BD Biosciences, cat. 610920), rabbit CDH1 antibody (Cell Signaling Technology, cat. 3195S), rabbit Arkadia antibody (Proteintech, cat. 14698-1-AP), mouse Ub (P4D1) antibody (Santa Cruz, SC-8017), rabbit MTSS1 antibody (CST, cat. 4385s), goat anti-rabbit IgG (Merck) and goat anti-mouse IgG (Merck) antibodies were used for western blot analyses. The mouse CDH2 antibody (BD Biosciences, cat. 610920), rabbit CDH1 antibody (Cell Signaling Technology), mouse VIM antibody (BD Biosciences, cat. 550513) and the Tyramide Signal Amplification kit (Shanghai EU-BIO, cat. EUT0168) were used for immunofluorescence analyses. Antibody dilutions were 1:500 for primary antibodies and 1:2,000 for secondary antibodies in immunohistochemistry analyses, 1:1,000 for primary antibodies and 1:5,000 for secondary antibodies in western blotting, and 1:200 for primary antibodies in immunofluorescence analyses. The biotinylated horse anti-mouse IgG antibody (Vector Labs) was used for immunohistochemistry analyses. The anti-miR-182 oligonucleotide ( $5^{\prime}$-AGUG UGAGUUCUACCAUUGCCAAA- $3^{\prime}$ ) and scrambled control oligonucleotide ( $5^{\prime}$-CAGUACUUUUGUGUAGUACAA- $3^{\prime}$ ) were previously reported ${ }^{23}$ and purchased from GenePharma (Shanghai, China). SMAD7 siRNA (5'-AGGUCAC CACCAUCCCCACTT- $\left.3^{\prime}\right)$ and the scrambled control (5'-UUCUCCGAACGUGU CACGUTT- $3^{\prime}$ ) were purchased from GenePharma. The DIG-labeled miRCURY LAN detection probes (Exiqon, 38489-01) and anti-Digoxigenin-POD, Fab fragments (Roche, cat. 11207733910) were used for in situ hybridization of miR182. MG-132 (Beyotime, s174) was used to inhibit the proteasome activity. The concentration for recombinant human TGF $\beta 1$ (R\&D) stimulation was $20 \mathrm{ng} \mathrm{ml}^{-1}$. The duration for TGF $\beta$ and MG-132 treatments was $48 \mathrm{~h}$ unless specified.

miRNA detection. Total RNA was extracted using TRIzol (Invitrogen). Mature miRNAs were reverse-transcribed and quantitated with the TaqMan
microRNA Assays (Applied Biosystems). The data was normalized to U6 expression.

Luciferase dual-reporter assays. HeLa cells were co-transfected with the miR-182-expressing plasmid or control vector, the indicated firefly luciferase reporter plasmids and a renilla luciferase plasmid with a ratio of 2:2:1. Lysates were collected $72 \mathrm{~h}$ after transfection. Firefly and renilla luciferase activities were measured with a Dual-Luciferase Reporter System (Promega).

Invasion assays. Cancer cells of $5 \times 10^{4}-10^{5}$ in serum-free medium were seeded into the upper chamber of transwell insert membranes of an $8 \mu \mathrm{m}$ pore size (Corning) coated with Matrigel (BD Biosciences) in a 24-well plate. Approximately $10 \%$ fetal bovine serum (FBS) was used in the bottom chamber as the attractant The cells in the upper chamber were removed 16-96 h later using a cotton swab. The invaded cells at the lower chamber were stained with crystal violet and counted. For TGF $\beta 1$ treatment, the cytokine $\left(20 \mathrm{ng} \mathrm{ml}^{-1}\right)$ was added into the upper chamber before the transwell invasion analysis.

Immunofluorescence analysis. Cancer cells of $\sim 30 \%$ confluence were fixed with PBS containing 3.7\% paraformaldehyde without methanol. The cells were washed and permeabilized with $0.2 \%$ Triton X-100 and blocked with $3 \%$ FBS for 30 min at room temperature. Then immunofluorescence staining was performed with the Tyramide Signal Amplification kit following manufacturer's protocol. Briefly, samples were incubated with the primary antibody overnight at $4{ }^{\circ} \mathrm{C}$, and then were treated with $4 \% \mathrm{H}_{2} \mathrm{O}_{2}$ for $15 \mathrm{~min}$, followed by incubation with POD-conjugated anti-mouse or anti-rabbit IgG for $1 \mathrm{~h}$ at room temperature. Following washing, samples were incubated with Rhodamine-conjugated tyramide for 15 min. 4,6-diamidino-2-phenylindole (DAPI) was used to counter-stain the nuclei. Pictures were visualized by three-dimensional confocal microscopy (LSM-510META, Carl Zeiss) and acquisition parameters were kept constant for all of the experiments.

Flow cytometry analysis. Cell apoptosis was determined by flow cytometry analysis. Cells were cultured in the absence or presence of $20 \mathrm{ng} \mathrm{ml}^{-1}$ TGF $\beta 1$ for 24 h. Cells were collected, washed with cold PBS, fixed in cold 70\% Ethanol, treated with DNase-free RNase (Sangon, RB473. $100 \mu \mathrm{g} \mathrm{ml}^{-1}$ ) and stained with $50 \mu \mathrm{g} \mathrm{ml}^{-1}$ Propidium iodide (Sangon, cat. P1112) and Annexin V-APC/7-AAD kit (KeyGEN, cat.KGA-1025). The cells were analysed using a Gallios flow cytometer (Beckman Coulter) to quantify the proportion of cells in apoptosis status

Animal experiments. To study primary tumour growth and lung metastasis $10^{5}$ 4T1 cancer cells were resuspended in 1:1 PBS and matrigel mixture and injected into the fat pads of fourth mammary glands of female BALB/c mice. Tumour growth was monitored twice a week by size measurement. Both maximum (L) and minimum (W) diameters of the tumours were measured using a slide caliper, and the tumour volume was calculated as $\pi \mathrm{LW}^{2} / 6$. The tumours were surgically removed when reaching the size of $1 \mathrm{~cm}$, and fixed by $4 \%$ paraformaldehyde for histological staining to analyse the local invasion. For lung metastasis analysis, all the mice were euthanized after 5 weeks post transplantation, and the lungs were fixed with Bouin's fixative. Then the metastasis nodules on the lung surfaces were counted. Ten mice per group were used for the above analyses

To study bone metastasis, $10^{5}$ SCP 28 cells were intracardially or intratibially injected to female nude mice. Bioluminescent imaging was acquired with a NightWOL II LB 983 Imaging System (Berthold). Bone damages were detected by $\mathrm{X}$-ray radiography with a Faxitron instrument (Faxitron Bioptics) as previously described_ENREF_9. Osteolytic areas were identified on radiographs as demarcated radiolucent lesions in bone and quantified using ImageJ. Forelimb and hindlimb long bone of mice were excised, fixed in $10 \%$ neutral-buffered formalin after $24 \mathrm{~h}$, decalcified (10\% EDTA, 2 weeks), dehydrated through a graded alcohol series, embedded in paraffin and stained with H\&E. All animal experiments were performed according to the guidelines for the care and use of laboratory animals and were approved by the Institutional Biomedical Research Ethics Committee of Shanghai Institutes for Biological Sciences.

Osteoclastogenesis assays. Primary bone marrow osteoclastogenesis analysis was performed with bone marrow from 4-to-6-week-old BALB/c mice. Bone marrow cells were harvested and plated in basal culture medium overnight. The next day, non-adherent cells were added at $5 \times 10^{5}$ per well to 24 -well plates that were previously seeded with either control or indicated tumour cells supplemented with $25 \mathrm{ng} \mathrm{ml}^{-1}$ RANKL(PeproTech) and $25 \mathrm{ng} \mathrm{ml}^{-1} \mathrm{M}$-CSF(PeproTech). Medium was changed every 3 days. Cancer cells were treated with $10 \mathrm{ng} \mathrm{ml}^{-1}$ TGF $\beta$ for $72 \mathrm{~h}$, and the resultant conditioned medium were mixed with $\alpha$-MEM at 1:9 ratio for bone marrow culturing. TRAP staining was performed with a TRAP kit (387A, Sigma-Aldrich) on day 10-12. Osteoclasts were defined as TRAPpositive cells containing more than three nuclei. 
Clinical samples and analyses. Fresh-frozen and paraffin-embedded tumour specimens were obtained Qilu Hospital of Shandong University with informed consent from all subjects and approval from the Institutional Research Ethics Committee. RNA was extracted from the fresh-frozen tumours and the quality was monitored by O.D. reading. miR-182 and SMAD7 RNA levels were measured by qPCR.

Paraffin-embedded tumour specimens were cut at a thickness of $5 \mu \mathrm{m}$. Then the sections were deparaffinized by Xylene and dehydrated through a graded alcohol series. The sections were incubated for $20 \mathrm{~min}$ in $3 \%$ hydrogen peroxide blocking solution and probed with the SMAD7 antibody (1:125) or the VIM antibody (1:200). Following extensive washings, sections were incubated for $1 \mathrm{~h}$ in the secondary antibody (1:200). Following washings, the Avidin Biotin Complex (Vector Labs) was then applied to the sections, followed by extensive washing steps. The DAB Peroxidase Substrate Kit (Vector Labs) was then added to the sections, and incubated for 2-3 min. Sections were then counterstained in hematoxylin (Sigma) and dehydrated in ascending grades of methanol before clearing in xylene and mounting under a cover slip. Finally each sample was scored as negative (0), low (1), medium (2) or high (3) according to staining intensities.

For miR-182 in situ hybridization, OCT-embedded fresh-frozen specimens were cut at a thickness of $10 \mu \mathrm{m}$ and fixed in $10 \%$ neutral-buffered formalin at room temperature overnight. The sections were treated with Proteinase $\mathrm{K}$ $\left(15 \mu \mathrm{g} \mathrm{ml}^{-1}\right)$ at $37^{\circ} \mathrm{C}$ for $15 \mathrm{~min}$, and incubated in the hybridization mix containing the miR-182 probe at $56^{\circ} \mathrm{C}$ for $1 \mathrm{~h}$. Following stringent washing in SSC, the slides were incubated with 3\% FBS as blocking solution for $30 \mathrm{~min}$ at room temperature. The sections were treated with $3 \% \mathrm{H}_{2} \mathrm{O}_{2}$ for $15 \mathrm{~min}$ in order to quench the endogenous peroxidase. Then the sections were applied with anti-DIG-POD $(1: 1,000)$. Following washings the DAB peroxidase substrate was applied to the sections for $5 \mathrm{~min}$. Sections were then counter-stained in hematoxylin and mounted under a cover slip.

TCGA data analyses. The analysis of TCGA miR-182 expression data in breast invasive carcinoma and normal tissues was based upon data generated by the TCGA Research Network: http://cancergenome.nih.gov. For metastasis-free survival analysis, patient clinical information was retrieved from the TCGA database. The median miR-182 expression level of the whole cohort was used as the cutoff to stratify all patients or the patients of luminal or triple negative subtypes into two groups with high or low expression of miR-182. The non-survival event was defined as distant metastasis or death. Survival probability of the two patient groups were compared with Kaplan-Meier curve. The HER2 + subtype was not analysed due the small number of patients.

Statistical analysis. Two-sided independent student's $t$-test without assumption of equal variance was performed to analyse the results of in vitro assays, animal experiments and clinical samples. Error bars in figures represent standard deviation of the experimental repeats. Quantification of western blots and immunohistochemistry analyses were performed with ImageJ. The band intensities of analysed proteins of western blots were normalized to that of GAPDH. The quantification data of western blots and immunohistochemistry can be found in Supplementary Fig. 12. The original scans of immuno-blots can be found in Supplementary Fig. 13.

Data availability. All relevant data supporting the findings of this study are available within the article, in Supplementary Information, or from the authors on request.

\section{References}

1. Massague, J. TGFbeta in cancer. Cell 134, 215-230 (2008)

2. Shi, Y. \& Massague, J. Mechanisms of TGF-beta signaling from cell membrane to the nucleus. Cell 113, 685-700 (2003).

3. Lim, J. \& Thiery, J. P. Epithelial-mesenchymal transitions: insights from development. Development 139, 3471-3486 (2012).

4. Shirkoohi, R. Epithelial mesenchymal transition from a natural gestational orchestration to a bizarre cancer disturbance. Cancer Sci. 104, 28-35 (2013).

5. Heldin, C. H., Vanlandewijck, M. \& Moustakas, A. Regulation of EMT by TGFbeta in cancer. FEBS Lett. 586, 1959-1970 (2012).

6. Kang, Y. \& Massague, J. Epithelial-mesenchymal transitions: twist in development and metastasis. Cell 118, 277-279 (2004).

7. Yang, J. et al. Twist, a master regulator of morphogenesis, plays an essential role in tumor metastasis. Cell 117, 927-939 (2004).

8. Mani, S. A. et al. The epithelial-mesenchymal transition generates cells with properties of stem cells. Cell 133, 704-715 (2008).

9. Wang, Y. et al. DLC1-dependent parathyroid hormone-like hormone inhibition suppresses breast cancer bone metastasis. J. Clin. Invest. 124, 1646-1659 (2014).

10. Ell, B. \& Kang, Y. SnapShot: Bone Metastasis. Cell 151, 690-690 e691 (2012).

11. Kang, Y. et al. Breast cancer bone metastasis mediated by the Smad tumor suppressor pathway. Proc. Natl Acad. Sci. USA 102, 13909-13914 (2005).
12. Lu, X. et al. In vivo dynamics and distinct functions of hypoxia in primary tumor growth and organotropic metastasis of breast cancer. Cancer Res. 70, 3905-3914 (2010).

13. Massague, J. \& Chen, Y. G. Controlling TGF-beta signaling. Genes Dev. 14, 627-644 (2000).

14. Kavsak, P. et al. Smad7 binds to Smurf2 to form an E3 ubiquitin ligase that targets the TGF beta receptor for degradation. Mol. Cell 6, 1365-1375 (2000)

15. Zhang, S. et al. Smad7 antagonizes transforming growth factor beta signaling in the nucleus by interfering with functional Smad-DNA complex formation. Mol. Cell Biol. 27, 4488-4499 (2007).

16. Afrakhte, M. et al. Induction of inhibitory Smad6 and Smad7 mRNA by TGFbeta family members. Biochem. Biophys. Res. Commun. 249, 505-511 (1998).

17. Zhang, J. \& Ma, L. MicroRNA control of epithelial-mesenchymal transition and metastasis. Cancer Metastasis Rev. 31, 653-662 (2012).

18. Butz, H., Racz, K., Hunyady, L. \& Patocs, A. Crosstalk between TGF-beta signaling and the microRNA machinery. Trends Pharmacol. Sci. 33, 382-393 (2012).

19. Song, L. et al. TGF-beta induces miR-182 to sustain NF-kappaB activation in glioma subsets. J. Clin. Invest. 122, 3563-3578 (2012).

20. Kang, Y. et al. A multigenic program mediating breast cancer metastasis to bone. Cancer Cell 3, 537-549 (2003).

21. Friedman, R. C., Farh, K. K., Burge, C. B. \& Bartel, D. P. Most mammalian mRNAs are conserved targets of microRNAs. Genome Res. 19, 92-105 (2009).

22. Lewis, B. P., Burge, C. B. \& Bartel, D. P. Conserved seed pairing, often flanked by adenosines, indicates that thousands of human genes are microRNA targets. Cell 120, 15-20 (2005).

23. Lei, R. et al. Suppression of MIM by microRNA-182 activates RhoA and promotes breast cancer metastasis. Oncogene 33, 1287-1296 (2014).

24. Koinuma, D. et al. Arkadia amplifies TGF-beta superfamily signalling through degradation of Smad7. EMBO J. 22, 6458-6470 (2003).

25. Qiu, Y. et al. TGF-beta upregulates miR-182 expression to promote gallbladder cancer metastasis by targeting CADM1. Mol. Biosyst. 10, 679-685 (2014).

26. Roodman, G. D. Genes associate with abnormal bone cell activity in bone metastasis. Cancer Metastasis Rev. 31, 569-578 (2012).

27. Guise, T. A. et al. Evidence for a causal role of parathyroid hormone-related protein in the pathogenesis of human breast cancer-mediated osteolysis. J. Clin. Invest. 98, 1544-1549 (1996).

28. Lindemann, R. K., Ballschmieter, P., Nordheim, A. \& Dittmer, J. Transforming growth factor beta regulates parathyroid hormone-related protein expression in MDA-MB-231 breast cancer cells through a novel Smad/Ets synergism. J. Biol. Chem. 276, 46661-46670 (2001).

29. Nakao, A. et al. Identification of Smad7, a TGFbeta-inducible antagonist of TGF-beta signalling. Nature 389, 631-635 (1997).

30. Dong, C. et al. Deficient Smad7 expression: a putative molecular defect in scleroderma. Proc. Natl Acad. Sci. USA 99, 3908-3913 (2002).

31. Jungert, K. et al. Smad-Sp1 complexes mediate TGFbeta-induced early transcription of oncogenic Smad7 in pancreatic cancer cells. Carcinogenesis 27, 2392-2401 (2006).

32. Monteleone, G. et al. Blocking Smad7 restores TGF-betal signaling in chronic inflammatory bowel disease. J. Clin. Invest. 108, 601-609 (2001).

33. Kleeff, J. et al. The TGF-beta signaling inhibitor Smad7 enhances tumorigenicity in pancreatic cancer. Oncogene 18, 5363-5372 (1999).

34. Massague, J. TGFbeta signalling in context. Nat. Rev. Mol. Cell Biol. 13, 616-630 (2012).

35. Brodin, G., Ahgren, A., ten Dijke, P., Heldin, C. H. \& Heuchel, R. Efficient TGF-beta induction of the Smad7 gene requires cooperation between AP-1, Sp1, and Smad proteins on the mouse Smad7 promoter. J. Biol. Chem. 275, 29023-29030 (2000).

36. Denissova, N. G., Pouponnot, C., Long, J., He, D. \& Liu, F. Transforming growth factor beta -inducible independent binding of SMAD to the Smad7 promoter. Proc. Natl Acad. Sci. USA 97, 6397-6402 (2000).

37. von Gersdorff, G. et al. Smad3 and Smad4 mediate transcriptional activation of the human Smad7 promoter by transforming growth factor beta. J. Biol. Chem. 275, 11320-11326 (2000).

38. Edlund, S. et al. Transforming growth factor-beta1 (TGF-beta)-induced apoptosis of prostate cancer cells involves Smad7-dependent activation of p38 by TGF-beta-activated kinase 1 and mitogen-activated protein kinase kinase 3 . Mol. Biol. Cell 14, 529-544 (2003).

39. Azuma, H. et al. Effect of Smad7 expression on metastasis of mouse mammary carcinoma JygMC(A) cells. J. Natl Cancer Inst. 97, 1734-1746 (2005).

40. Liu, X. et al. Smad7 but not Smad6 cooperates with oncogenic ras to cause malignant conversion in a mouse model for squamous cell carcinoma. Cancer Res. 63, 7760-7768 (2003).

41. Javelaud, D. et al. Stable overexpression of Smad7 in human melanoma cells impairs bone metastasis. Cancer Res. 67, 2317-2324 (2007).

42. Halder, S. K., Rachakonda, G., Deane, N. G. \& Datta, P. K. Smad7 induces hepatic metastasis in colorectal cancer. Br. J. Cancer 99, 957-965 (2008). 
43. Hanahan, D. \& Weinberg, R. A. Hallmarks of cancer: the next generation. Cell 144, 646-674 (2011).

44. Jiang, X. et al. Genetic variations in SMAD7 are associated with colorectal cancer risk in the colon cancer family registry. PLoS ONE 8, e60464 (2013).

45. Boulay, J. L. et al. SMAD7 is a prognostic marker in patients with colorectal cancer. Int. J. Cancer 104, 446-449 (2003).

46. Hariharan, R., Babu, J. M, P, R. \& Pillai, M. R. Mutational analysis of Smad7 in human cervical cancer. Oncol. Rep. 21, 1001-1004 (2009).

47. Wang, D., Kanuma, T., Mizumuma, H., Ibuki, Y. \& Takenoshita, S. Mutation analysis of the Smad6 and Smad7 gene in human ovarian cancers. Int. J. Oncol. 17, 1087-1091 (2000).

48. Nagarajan, R. P., Zhang, J., Li, W. \& Chen, Y. Regulation of Smad7 promoter by direct association with Smad3 and Smad4. J. Biol. Chem. 274, 33412-33418 (1999).

49. Bauge, C. et al. Interleukin-1beta up-regulation of Smad7 via NF-kappaB activation in human chondrocytes. Arthritis Rheum. 58, 221-226 (2008).

50. Monteleone, G. et al. Post-transcriptional regulation of Smad7 in the gut of patients with inflammatory bowel disease. Gastroenterology 129, 1420-1429 (2005).

51. Zhang, L. et al. RNF12 controls embryonic stem cell fate and morphogenesis in zebrafish embryos by targeting Smad7 for degradation. Mol. Cell 46, 650-661 (2012).

52. Liu, G. et al. miR-21 mediates fibrogenic activation of pulmonary fibroblasts and lung fibrosis. J. Exp. Med. 207, 1589-1597 (2010).

53. Smith, A. L. et al. The miR-106b-25 cluster targets Smad7, activates TGF-beta signaling, and induces EMT and tumor initiating cell characteristics downstream of Sixl in human breast cancer. Oncogene 31, 5162-5171 (2012).

54. Xia, H., Ooi, L. L. \& Hui, K. M. MicroRNA-216a/217-induced epithelialmesenchymal transition targets PTEN and SMAD7 to promote drug resistance and recurrence of liver cancer. Hepatology 58, 629-641 (2013).

55. Singh, P., Wig, J. D., Srinivasan, R. \& Radotra, B. D. A comprehensive examination of Smad4, Smad6 and Smad7 mRNA expression in pancreatic ductal adenocarcinoma. Indian J. Cancer 48, 170-174 (2011).

56. Huang, Q. et al. Expression of Smad7 in cholangiocarcinoma: prognostic significance and implications for tumor metastasis. Asian. Pac. J. Cancer Prev. 13, 5161-5165 (2012).

57. Wang, P. et al. Low-level expression of Smad7 correlates with lymph node metastasis and poor prognosis in patients with pancreatic cancer. Ann. Surg. Oncol. 16, 826-835 (2009).

58. Osawa, H., Nakajima, M., Kato, H., Fukuchi, M. \& Kuwano, H. Prognostic value of the expression of Smad6 and Smad7, as inhibitory Smads of the TGFbeta superfamily, in esophageal squamous cell carcinoma. Anticancer Res. 24, 3703-3709 (2004).

59. Mangone, F. R. et al. Smad2 and Smad6 as predictors of overall survival in oral squamous cell carcinoma patients. Mol. Cancer. 9, 106 (2010).

60. Puhringer-Oppermann, F., Sarbia, M., Ott, N. \& Brucher, B. L. The predictive value of genes of the TGF-beta1 pathway in multimodally treated squamous cell carcinoma of the esophagus. Int. J. Colorectal. Dis. 25, 515-521 (2010).

61. Xu, S., Witmer, P. D., Lumayag, S., Kovacs, B. \& Valle, D. MicroRNA (miRNA) transcriptome of mouse retina and identification of a sensory organ-specific miRNA cluster. J. Biol. Chem. 282, 25053-25066 (2007).

62. Moskwa, P. et al. miR-182-mediated downregulation of BRCA1 impacts DNA repair and sensitivity to PARP inhibitors. Mol. Cell 41, 210-220 (2011).

63. Stittrich, A. B. et al. The microRNA miR-182 is induced by IL-2 and promotes clonal expansion of activated helper T lymphocytes. Nat. Immunol. 11, 1057-1062 (2010).

64. Saus, E. et al. Genetic variants and abnormal processing of pre-miR-182, a circadian clock modulator, in major depression patients with late insomnia. Hum. Mol. Genet. 19, 4017-4025 (2010).
65. Segura, M. F. et al. Aberrant miR-182 expression promotes melanoma metastasis by repressing FOXO3 and microphthalmia-associated transcription factor. Proc. Natl Acad. Sci. USA 106, 1814-1819 (2009).

66. Jiang, L. et al. miR-182 as a prognostic marker for glioma progression and patient survival. Am. J. Pathol. 177, 29-38 (2010).

67. Liu, Z. et al. MiR-182 overexpression in tumourigenesis of high-grade serous ovarian carcinoma. J. Pathol. 228, 204-215 (2012).

68. Li, X. L. et al. A p21-ZEB1 complex inhibits epithelial-mesenchymal transition through the miR-183-96-182 cluster. Mol. Cell Biol. 3, 533-550 (2013).

69. Qu, Y. et al. MiR-182 and miR-203 induce mesenchymal to epithelial transition and self-sufficiency of growth signals via repressing SNAI2 in prostate cells. Int. J. Cancer 133, 544-555 (2013).

70. Strickland, L. B., Dawson, P. J., Santner, S. J. \& Miller, F. R. Progression of premalignant MCF10AT generates heterogeneous malignant variants with characteristic histologic types and immunohistochemical markers. Breast Cancer Res. Treat. 64, 235-240 (2000).

\section{Acknowledgements}

We thank Dr Y. Kang for providing the SMAD4 overexpression plasmid, Dr C-H. Heldin for the SMAD7 overexpression plasmid and L. Fu, S. Yan and P. Zhou at the Institute of Health Sciences core facilities for technical support. The study was funded by grants from the National Natural Science Foundation of China $(81430070,31371409,81222032)$, the Ministry of Science and Technology of China (2012ZX09506-001-005, 2013CB910904) Chinese Academy of Sciences (QYZDB-SSW-SMC013, XDA12050101, XDA01040402) and Science and Technology Commission of Shanghai Municipality (14431900800).

\section{Author contributions}

G.H. supervised the work. J.Y., R.L. and G.H. designed the experiments and drafted the manuscript. J.Y., R.L., X.Z., X.L., G.L. and X.Z. performed the experiments. S.L. and M.F.S. helped design the experiments and critically reviewed the manuscript. All authors discussed the results and commented on the manuscript.

\section{Additional information}

Supplementary Information accompanies this paper at http://www.nature.com/ naturecommunications

Competing financial interests: The authors declare no competing financial interests.

Reprints and permission information is available online at http://npg.nature.com/ reprintsandpermissions/

How to cite this article: Yu, J. et al. MicroRNA-182 targets SMAD7 to potentiate TGF $\beta$-induced epithelial-mesenchymal transition and metastasis of cancer cells. Nat. Commun. 7, 13884 doi: 10.1038/ncomms13884 (2016).

Publisher's note: Springer Nature remains neutral with regard to jurisdictional claims in published maps and institutional affiliations.

This work is licensed under a Creative Commons Attribution 4.0 International License. The images or other third party material in this (c) (i) ded in the article's Creative Commons license, unless indicated otherwise article are included if the material is not included under the Creative Commons license, users will need to obtain permission from the license holder to reproduce the material. To view a copy of this license, visit http://creativecommons.org/licenses/by/4.0/

(C) The Author(s) 2016 